\title{
REVIEW
}

\section{Prevention of hepatocellular carcinoma in nonviral-related liver diseases}

\author{
Jian-Gao Fan, ${ }^{*}{ }^{\dagger}$ Geoffrey C Farrell ${ }^{\dagger}$ and the Asia-Pacific Working Party for Prevention of Hepatocellular \\ Carcinoma \\ *Department of Gastroenterology, Xinhua Hospital, Shanghai Jiaotong University School of Medicine, Shanghai, China; and ${ }^{\dagger}$ Gastroenterology and \\ Hepatology Unit, The Canberra Hospital and Australian National University Medical School, Canberra, ACT, Australia
}

\author{
Key words \\ aflatoxin, alcoholism, cirrhosis, diabetes, \\ hereditary hemochromatosis, non-alcoholic \\ fatty liver disease, obesity. \\ Accepted for publication 2 December 2008.

\section{Correspondence} \\ Professor Jian-Gao Fan, Visiting scholar of \\ ANU Medical School and Director of \\ Department of Gastroenterology, Xinhua \\ Hospital, Shanghai Jiaotong University School \\ of Medicine, Shanghai 200092, China. Email: \\ fanjiangao@gmail.com
}

\begin{abstract}
Although chronic infection with hepatitis $\mathrm{B}$ virus and/or hepatitis $\mathrm{C}$ virus are the most important risk factors for hepatocellular carcinoma (HCC) worldwide, other causes of cirrhosis can also lead to HCC. Given the high prevalence of alcoholism and the worldwide obesity epidemic, the relevant importance of nonviral liver disease-related HCC is expected to increase in the future. Some evidence supports mechanistic interactions between host or environmental factors and chronic viral hepatitis in the development of HCC. For example, food- and water-borne carcinogens have contributed to unusually high rates of $\mathrm{HCC}$ in parts of China and sub-Saharan Africa. With some of these conditions, appropriate public health measures to reduce the population's exposure to known etiologic agents, or early therapeutic intervention for 'at-risk' individuals before development of cirrhosis (e.g. hereditary hemochromatosis) can prevent HCC. Community-based programs to discourage and deal with excessive alcohol intake, to promote tobacco smoking awareness, to avoid exposure to aflatoxin and other food toxins, and measures to reduce the pandemic of obesity and diabetes are vital for effective interruption of the rising tide of HCC from nonviral liver disease.
\end{abstract}

\section{Introduction}

Hepatocellular carcinoma (HCC) is the fifth most common cancer worldwide and the third most common cause of cancer mortality. ${ }^{1-4}$ Despite major efforts to improve the diagnosis and treatment, therapeutic options for HCC have not impacted on the mortality. ${ }^{3-5}$ The global health burden of HCC can reasonably be reduced by early detection or prevention. ${ }^{3-7}$ Prevention of HCC is possible because, in more than $90 \%$ of cases, this cancer occurs in the context of known risk factors, most of which are theoretically preventable. ${ }^{8}$ The key, as reviewed in other articles of this series, the risk factors associated with $\mathrm{HCC}$ include chronic hepatitis $\mathrm{B}(\mathrm{CHB})$ and/or hepatitis $\mathrm{C}(\mathrm{CHC})$, particularly with subsequent cirrhosis. ${ }^{8-10}$ However, cirrhosis arising from nonviral liver diseases (Table 1) is also risk for HCC, such as alcoholic liver disease (ALD), non-alcoholic fatty liver disease (NAFLD) and non-alcoholic steatohepatitis (NASH), hereditary hemochromatosis $(\mathrm{HH})$ and, less commonly, drug- and toxin-induced, immune-mediated or metabolic liver diseases, that is, primary biliary cirrhosis (PBC) and $\alpha-1$ antitrypsin deficiency. ${ }^{2,8,11}$ With some of these conditions, appropriate public health measures to reduce the population's exposure to the known etiologic agents, or early therapeutic intervention for 'at-risk' individuals before development of cirrhosis could prevent HCC. Additional factors account for some regional or individual variability in HCC risk. Other host and environmental factors can therefore influence the development of HCC, even though they may not be necessary or sufficient for hepatocarcinogenesis when present alone. Some host factors include advanced age and male gender, excessive alcohol consumption, iron overload, cigarette smoking, obesity, and type 2 diabetes. ${ }^{1-4,8,11,12}$ Food- and water-borne carcinogens, such as aflatoxin B1 and fumonisin from contaminated crops, have contributed to unusually high rates of HCC in parts of China and sub-Saharan Africa. ${ }^{1,2,4,6}$ Clean water and uncontaminated food supplies offer an additional basic condition to prevent HCC.

Although CHB is the most common etiologic agent of HCC in the Asia-Pacific region, with the exception of Japan, where cases of $\mathrm{HCC}$ are mainly related to $\mathrm{CHC}$, and Australia and New Zealand where $\mathrm{CHB}$ and $\mathrm{CHC}$ are both important, nonviral liver diseases are implicated in $10-20 \%$ of cases. ${ }^{1-3,12-16}$ There is also possible interaction between host and environmental factors with chronic hepatitis B virus/hepatitis $\mathrm{C}$ virus (HBV/HCV) infection in the development of HCC. . $^{1-3,8,14}$

In this review we will focus on risk factors for $\mathrm{HCC}$ in patients without chronic viral hepatitis, with an emphasis on opportunities for preventing chronic liver disease and development of HCC as one of its complications. 
Table 1 Causes and risk factors for HCC and general approaches to prevention

\begin{tabular}{ll}
\hline Etiology or risk factors & Major approaches to prevention \\
\hline Major causes & \\
Chronic HBV infection & Vaccination; antiviral therapy \\
Chronic HCV infection & Screening of blood; antiviral therapy \\
Alcohol liver disease & Abstinence of alcohol intake; \\
& correction of nutritional \\
& deficiencies \\
Minor causes & \\
Non-alcoholic fatty liver & ${ }^{\dagger}$ Treating metabolic risk factors \\
disease & \\
Hereditary hemochromatosis & Family screening; iron depletion \\
Primary biliary cirrhosis & ${ }^{\dagger}$ Using ursodeoxycholic acid \\
Risk factors & \\
Dietary pollution & Avoiding exposure to aflatoxins \\
Alcoholism & ${ }^{\dagger}$ Screen for and treatment of \\
Overweight/obesity & alcohol dependence and abuse \\
Type 2 diabetes & ${ }^{\dagger}$ Diet and aerobic exercise \\
Tobacco smoking & ${ }^{\dagger}$ Optimal control of blood glucose \\
\hline
\end{tabular}

'These approaches have not yet been shown to be effective. $\mathrm{HBV}$, hepatitis B virus; HCC, hepatocellular carcinoma; HCV, hepatitis C virus.

\section{Risk factors for HCC in the absence of HBV or HCV infection}

\section{Cirrhosis}

With rare exceptions, HCC is a complication of chronic liver disease, usually at the stage of cirrhosis. ${ }^{12,17}$ Cirrhosis of the liver can therefore be regarded as a premalignant condition independent of its etiology. ${ }^{1,17}$ More than $80 \%$ of HCC developed in a cirrhotic liver, and $\mathrm{HCC}$ is the leading cause of death (or roughly equal to liver failure) in patients with cirrhosis.,12,17 The annual risk of developing HCC is between $1 \%$ and $6 \%$. The risk to develop this malignancy depends on the necroinflammatory activity of the underlying hepatitis, its etiology, and the duration of the disease. ${ }^{8,17}$ Patients suffering from cirrhosis because of chronic HBV, $\mathrm{HCV}$, or hepatitis D virus infection and those with $\mathrm{HH}$ exhibit a high risk for HCC. This risk is further increased by exposure to co-carcinogens, such as alcohol, cigarette smoke, and dietary aflatoxins. Cirrhosis resulting from multiple risk factors may be associated with an increased incidence of HCC..$^{8,17,18}$ However, development of HCC in patients with cirrhosis because of autoimmune hepatitis, Wilson's disease, and $\alpha-1$ antitrypsin deficiency is rare, and with $\mathrm{PBC}$ it is uncommon except in older men with cirrhosis. ${ }^{17-23}$

Although the incidence of HCC is increasing in lowprevalence regions such as Australian and North America, the relationship of this increase to individual risk factors remains unclear. A significant proportion of HCC cases (7-30\%) develop in 'cryptogenic cirrhosis', and this condition could represent the most worrisome complication of NASH, at a stage when the characteristic pathological features of steatosis and necroinflammation are no longer conspicuous. ${ }^{24-27}$ The rising prevalence of risk factors associated with NASH could account for the appar- ent increasing incidence of HCC complicating cryptogenic cirrhosis, but prospective epidemiological studies are required to clarify this. Occult HBV infection (serum HBsAg not detectable, anti-HBc positive, HBV-DNA extracted from the liver) and distant past history of excessive alcohol exposure might also play important roles in hepatocarcinogenesis in some individuals with 'cryptogenic cirrhosis'.

Irrespective of the above considerations the duration of cirrhosis seems to be an important factor for HCC, regardless of cause. $^{12,17-19,28}$ Thus there is a steady increase in HCC incidence from early cirrhosis without portal hypertension to advanced cirrhosis with hepatic compensation (Child-Pugh class C). The prevalence of HCC among unscreened patients with compensated cirrhosis has been reported as $5 \%$, and this percentage increases to $15-20 \%$ among patients with variceal bleeding or bacterial peritonitis. ${ }^{28}$

Older age is a consistent predictor of HCC; the relative importance of age as a surrogate marker of disease duration versus biologic effects on liver is unclear. ${ }^{8,17}$ Male sex is another consistent marker of increased HCC risk. ${ }^{8,17}$ It seems likely that this reflects operation of biologic factors because the same phenomenon is observed in mice, at least partly related to androgenic steroids, but it is salient to note that men also have higher exposure to co-carcinogens, such as alcohol and cigarette smoke. ${ }^{1,8}$

\section{Alcoholism and related liver disease}

Alcoholism is a worldwide problem among people of all ethnic and socioeconomic groups. ${ }^{29-31}$ Chronic excessive alcohol consumption is a strong risk factor for several cancers, but particularly HCC. ${ }^{14,32-34}$ More than 18 million adults in the USA abuse alcohol, a prevalence of $6 \%$, which is five times higher than that of hepatitis C. ${ }^{29,32-34}$ Alcohol will continue to be a common cause of HCC, as alcoholism increasing in many countries. ${ }^{29,32-34}$ The prevalence of problem drinking is not always discussed opening in countries where alcohol is a factor, including many populous Asian countries.

There is continuous dose-effect curve without a definite threshold between alcohol intake and the risk for HCC. However, most studies have found that HCC risk increases only for alcohol consumption above 40-60 g/day. ${ }^{29,32-34}$ Chronic alcohol use of greater than $80 \mathrm{~g} /$ day for more than 10 years increases the risk for HCC about fivefold, but lifetime alcohol exposure may be even more important. ${ }^{29,32-34}$ The risk of HCC is further increased if cirrhosis is present. A role for alcohol as a 'pure' hepatocarcinogen in the absence of cirrhosis seems of minor importance. ${ }^{29,32-34}$ Further, the incidence of HCC among patients with decompensated alcoholic cirrhosis does not decrease with abstinence from alcohol. In both genders, the risk may even increase further up to 10 years after stopping alcohol drinking. ${ }^{32}$

In addition, there is some evidence to support interactions between alcohol intake and $\mathrm{HCV}$ infection, and possibly with HBV infection, for risk of HCC. Thus, alcohol use in CHC doubles risk for HCC compared with $\mathrm{CHC}$ alone. Further, among HCV-infected persons who abuse alcohol HCC may occur at an earlier age and be histologically more advanced. ${ }^{34}$ Studies from east Asia have failed to find an interaction between habitual drinking and chronic HBV infection on HCC risk. Conversely, interactions between alcohol, obesity, and diabetes on 
development of cirrhosis and risk for HCC have been observed in case-control studies. ${ }^{14,35}$

The mechanisms by which alcohol contributes to HCC risk are not known. Hypothesized pathways include oxidative stress, changes in retinoic acid metabolism and DNA methylation, decreased immune surveillance, and genetic susceptibility. ${ }^{32-35}$

\section{Obesity, type 2 diabetes, and NAFLD}

Over the past few decades, the proportion of populations that are overweight or obese has increased substantially. ${ }^{36-38}$ This increase is undoubtedly related to changes in lifestyle that affect diet and physical activity. Today, about half the adult population in developed countries is overweight or obese, and urban populations of many developing countries are approaching similar proportions. Obesity is a major preventable cause of illness and premature death and contributes to mortality and the overall burden of disease worldwide. ${ }^{37,38}$ In addition to the risk of type 2 diabetes, NAFLD and cardiovascular disease, increased body mass index (BMI) seems to be an important risk factor for some cancers, including liver cancer. ${ }^{37-39}$ Obesity represents an independent risk factor for HCC in patients who have cryptogenic cirrhosis or alcoholic cirrhosis, while the risk of $\mathrm{HCC}$ in patients with $\mathrm{CHC}$ appears to increase in proportion to BMI across a wide range of values, from underweight to obese..$^{24-27}$

Type 2 diabetes is an independent risk factor for $\mathrm{HCC}$, irrespective of the primary cause. ${ }^{40-42}$ The increased incidence of HCC among those with diabetes has ranged from twofold to fourfold in different studies with varying ethnic cohorts. Even after stratifying for age, gender, ethnicity, viral hepatitis, HH, and ALD, diabetes increases HCC risk twofold to threefold. ${ }^{40,41}$ Diabetes appears to increase the odds of developing HCC through synergistic actions with other variables, such as viral hepatitis and alcohol. ${ }^{14}$ In addition, coexistent diabetes appears to increase the recurrence of HCC after potentially curative therapy, regardless of the etiology of liver disease. ${ }^{42}$

The most common liver disease seen in patients with obesity and/or diabetes is NAFLD. ${ }^{39,43-45}$ The clinicopathological spectrum ranges from simple steatosis to NASH, and many cases of cryptogenic cirrhosis occur in association with obesity and diabetes. ${ }^{45}$ Simple steatosis has a relatively benign clinical course, but NASH can progress to cirrhosis and HCC, although it appears to do so less frequency than for $\mathrm{CHC}^{26,44,45}$ The steadily increasing prevalence of obesity, coupled with diabetes and the metabolic syndrome has put a very large proportion of the population at risk of developing NASH and related cirrhosis, liver failure, and HCC. ${ }^{25,38,39,44,45}$ It is therefore possible that the current pandemic of obesity and diabetes has contributed to the doubling of HCC incidence in the USA and Australia during the past two decades.

The evidence that HCC is part of the natural history of NASH comes from three lines of research: (i) retrospective studies linking $\mathrm{HCC}$ arising in cryptogenic cirrhosis to risk factors for NASH; (ii) case reports; and (iii) prospective studies assessing the long-term complications of NASH or of NASH-related cirrhosis. As for other disorders, older men may be at higher risk. The relative roles of cirrhosis, steatohepatitis, and the underlying metabolic derangements in hepatocarcinogenesis need to be elucidated. ${ }^{25}$

\section{Iron overload and hemochromatosis}

Hereditary hemochromatosis is an autosomal recessive disorder. In some populations, those of Anglo-Celtic and Nordic origin, the homozygosity frequency of the most common mutation $\mathrm{C} 282 \mathrm{Y}$ is as high as one in 220 to one in $400 .{ }^{46} \mathrm{HCC}$ occurs predominantly in patients with cirrhosis at the time of diagnosis, although the presence of cirrhosis is not an absolute prerequisite. ${ }^{46-49}$ At the stage of cirrhosis, HCC is the most common cause of death from $\mathrm{HH}$. Venesection before development of cirrhosis prevents hepatic fibrosis and is associated with normal life expectancy. However, once cirrhosis is established the risk of HCC is not abolished by phlebotomy. ${ }^{47,48}$

The risk for HCC is also increased among African Black people with dietary iron overload and possibly in other ironloading disorders such as homozygous $\beta$ thalassemia. ${ }^{50}$ Dietary iron overload occurs commonly in parts of sub-Saharan Africa. It results from the consumption of large volumes of traditional beer that is home-brewed in iron pots or drums and consequently has higher iron content. The liver becomes iron-overloaded and may develop portal fibrosis or, less often, cirrhosis. ${ }^{50}$ Mildly to moderately increasing hepatic iron stores or HFE mutations are also associated independently with an increased risk for HCC among patients with other types of liver disease, suggesting a possible carcinogenic or co-carcinogenic role for iron in chronic liver disease. ${ }^{47,51}$ The mechanisms of iron-induced malignant transformation are yet to be fully characterized, but the close association between cirrhosis and HCC in patients with $\mathrm{HH}$, and the lesser association in those with dietary iron overload, suggests that chronic liver injury contributes to the malignant transformation, although iron may also be directly carcinogenic. ${ }^{46,47,51}$

\section{Prevention of nonviral liver disease-related HCC}

\section{Prevention and control of ALD}

\section{Reduction of alcohol consumption}

Recognition that hazardous drinking is linked to liver injury and risk of liver cancer should motivate individual counseling on health maintenance, as well as rational public health policies. A principal objective in the community is to reduce per capita alcohol consumption. Historically, prevention strategies have involved limiting availability, enforcement of stricter laws pertaining to alcohol use, education to improve community standards, and increasing the cost of alcoholic beverages through taxation. Measures that address the economic and physical accessibility of alcohol, particularly making it more expensive, appear to be the most effective..$^{3,29-32,52-54}$ Primary care providers can be asked to screen for and to treat alcohol dependence and abuse. Office screening tools in medical practice, combined with relatively brief interventions, can be powerful methods to assist those numerous individuals at risk for and those with ALD. To date, despite its undoubted importance as a risk factor, there is limited evidence to support the effectiveness of alcohol reduction interventions at reducing the incidence of HCC. . $^{3,29,52-54}$ 
Table 2 Efforts approach to reversal obesity epidemic

Increase education on diet and physical activity

Train health professionals

Restrict advertisements of high-calorie and low-nutrient foods

Limit access to unhealthy foods in schools and workplaces

Levy taxes on sugary drinks and other foods high in calories, fat, or sugar

Lower the prices of healthy foods

Promote physical activity in schools and workplaces

\section{Treatment of ALD}

Continued abstinence from alcohol consumption and correction of nutritional deficiencies are critical aspects for the long-term management of ALD. ${ }^{3,54}$ A survival benefit from abstinence has been established, but it is unclear the extent to which this reflects protection from liver failure and complications of portal hypertension and infection versus any protection from earlier carcinogenic effects of alcohol. Few studies have addressed whether continued alcohol intake is relevant to onset of HCC, or whether the risk decreases in those who achieve abstinence. ${ }^{3}$ In one study, the risk appeared to be the highest during the first 10 years after stopping excessive alcohol consumption. ${ }^{32}$ Further studies are required to establish this and to identify whether individual risk factors additional to the undoubted importance of age and male gender are important, particularly any factors that might be amenable to therapeutic intervention.

The treatment of alcoholic cirrhosis remains symptomatic, except for liver transplantation in advanced cases in which abstention from alcohol has been sustained for at least 6 months..$^{53}$ The risk for $\mathrm{HCC}$ in decompensated alcoholic cirrhosis approaches $1 \%$ per year, a risk that may not decrease with abstinence. ${ }^{3,32}$ Therefore, patients with end-stage ALD should be screened for HCC and considered for liver transplantation once abstinent for 6 months; the results are similar to those in non-alcoholic patients.

\section{Prevention and control of NAFLD}

\section{Control of overweight and obesity}

Reversal of the obesity epidemic will require multiple public health approaches: education, time planning, recreational facilities, and possible legislation to improve food choices. Communities, workplaces, schools, and medical centers are subject to federal and governmental regulations that could be modified to make the environment more conducive to healthy food choices and increased physical activity (Table 2). ${ }^{37,38}$

Currently available weight loss treatments include dietary intervention, particularly coupled to increased physical activity, behavior modification, pharmacotherapy, and bariatric surgery. Common behaviors among patients with obesity who have achieved successful long-term weight loss without bariatric surgery are a guide to cost-effective interventions (Table 3 ). ${ }^{3,37}$

\section{Treatment of NAFLD and cryptogenic cirrhosis}

Lifestyle modification that includes dietary restriction and exercise to achieve judicious weight loss, particularly that required to correct central obesity, coupled to appropriate management
Table 3 Behavior modification for patients with obesity

Consume a diet low in calories and fat

Regular engage in moderate physical activity

Check body weight and abdominal circumference regularly

of diabetes and dyslipidemia, is recommended as the first approach. ${ }^{55-57}$ Those with morbid obesity (BMI $>35 \mathrm{~kg} / \mathrm{m}^{2}$ in Europeans; possibly $>30 \mathrm{~kg} / \mathrm{m}^{2}$ in Asians) or obesity refractory to medical and lifestyle approaches should be considered for bariatric surgery. ${ }^{55-57}$ Other potential therapeutic modalities against NASH include insulin-sensitizing agents (metformin, rosiglitazone, and piaglitazone), anti-oxidants, cytoprotective agents, and lipidlowering drugs. However, these all remain investigational; none have been shown in large scale randomized controlled trials to be effective, and these approaches are currently not recommended for routine clinical practice. ${ }^{3,55-57}$

\section{Early detection and treatment of iron overload disorders}

\section{Screening and early detection}

Homozygosity of C282Y is readily detected by a simple molecular assay based on restriction fragment length polymorphisms. ${ }^{46,58-60}$ The high prevalence of the $\mathrm{C} 282 \mathrm{Y}$ mutation coupled with noninvasive diagnostic and simple efficient therapies represents arguments in favor of general screening of White people populations, or, more particularly, family screening after discovery of an affected proband. ${ }^{59-61}$ It has been recommended that screening for plasma transferrin saturation should be done first with subsequent genetic testing confined to those individuals with high transferrin saturation. However, the variable disease penetrance of $\mathrm{C} 282 \mathrm{Y}$ homozygosity and the limitation of transferrin saturation as an initial screening test make this view less appealing than more widespread application of C282Y gene testing. ${ }^{3,46,58-60}$

Disease penetrance for homozygous $\mathrm{C} 282 \mathrm{Y}$ individuals varies from $10 \%$ to $60 \%$. Those younger than 40 years with serum ferritin levels below $1000 \mathrm{ng} / \mathrm{mL}$ and normal serum alanine transaminase levels do not have advanced hepatic fibrosis or cirrhosis. Liver biopsy is therefore unnecessary, and screening for HCC is not required once venesection has been instituted. In other circumstances, liver biopsy is indicated to establish the state of hepatic fibrosis, the corresponding risk of HCC, and therefore the need for HCC screening. ${ }^{3,7}$ Currently, the combination of genetic testing and magnetic resonance imaging for diagnosing hepatic iron overload has drastically reduced the need for liver biopsy. ${ }^{46,59}$

\section{Reducing iron overload}

Venesection remains the standard treatment for $\mathrm{HH}$, non-HFE hemochromatosis, and acquired iron overload conditions unrelated to hematologic disorders. ${ }^{46,62}$ Timely and regular phlebotomy protects against development of cirrhosis, and thereby prevents onset of HCC. ${ }^{46,62}$ It is therefore important to identify persons with hemochromatosis gene defects before excessive iron stores cause tissue damage, and phlebotomy should be performed when there is evidence of iron accumulation, such as sustained elevation of 
serum ferritin and increased transferrin iron saturation. Once cirrhosis is established, the risk of HCC is not abolished by phlebotomy. Screening for HCC should be introduced. ${ }^{3,7}$

Reducing dietary intake of iron does not seem to be useful. Nutritional advice can only advocate a balanced diet with low alcohol intake, no excessive vitamin $\mathrm{C}$ supplementation (because this can reduce ferric iron to form ferrous iron, which is potentially cardiotoxic), and possibly, the consumption of tea (which chelates dietary iron). ${ }^{62,63}$

In forms of iron overload other than $\mathrm{HH}$ and dietary iron overload, phlebotomy is contraindicated. Iron chelators, including desferrioxamine, deferiprone, and deferasirox, are the treatment of choice for iron-loading anemias. The effects, if any, on HCC prevention have not yet been documented. ${ }^{63,64}$

\section{Treatment of cirrhosis because of other etiologies}

Although there is a risk of $\mathrm{HCC}$ in patients with stage IV PBC, particularly in men, it is a rare complication. ${ }^{21}$ Ursodeoxycholic acid (UDCA) is the only medication currently known to slow disease progression in PBC. ${ }^{64}$ Whereas, UDCA could lower incidence of HCC is unclear, but one recent systematic review failed to find evidence for any benefit of UDCA on mortality or rate of liver transplantation among patients with PBC..$^{65,66}$

\section{Prevention of sex steroid-related liver tumors}

Estrogens and androgenic steroids have both been related to development of liver tumors, most often benign, but sometimes HCC. This topic has been reviewed elsewhere, and there are few recent developments. ${ }^{67,68}$ Long-term high-dose estrogen-containing oral contraceptive steroids increase the risk of HCC, particularly the fibrolamellar variant that occurs in non-cirrhotic liver. Avoidance of continued oral contraceptive use without interruption, particularly with higher estrogen-containing preparations is now advised for a variety of health reasons, particularly thromboembolic and other vascular complications. Regular physical examination to detect hepatic enlargement or liver tumors, or annual ultrasound screening would be reasonable precautions if medical indications are such that prolonged exposure to high-dose estrogens is unavoidable. Similar arguments can be mounted for androgenic steroids. However, no effects, of oral contraceptive steroids on $\mathrm{HCC}$ risk could be detected in HBV-endemic countries.

\section{Other prevention measures to reduce incidence of HCC}

\section{Avoiding exposure to aflatoxins}

The aspergillus fungal toxin, aflatoxin B1, and particularly its epoxide metabolite, is a potent mutagen. Aflatoxin acts synergistically with HBV infection to amplify the risk of HCC. Aflatoxin contamination of the food supply occurs in tropical and subtropical humid areas, which coincides with high prevalence rates for $\mathrm{HBV}$ and HCC. ${ }^{4,8,69}$ Aflatoxin exposure in association with HBV infection induces a 60 -fold increase in risk of $\mathrm{HCC}$, while aflatoxin alone increases the risk fourfold. ${ }^{8,69,70}$ The marked worldwide heterogeneity of $\mathrm{HCC}$ incidence in $\mathrm{HBV}$-endemic regions might
Table 4 Avoiding dietary aflatoxin contamination

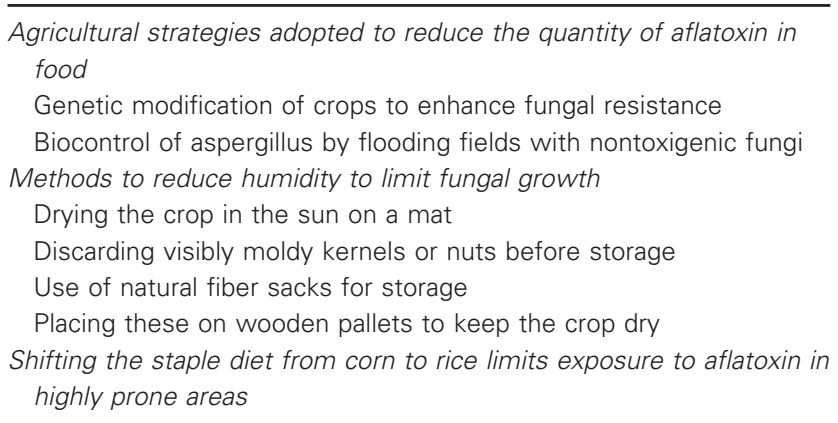

be related, at least in part, to aflatoxin exposure..$^{2,3,8}$ The mechanism involves mutation of tumor suppressor genes, particularly p53. The so-called p53 'mutation hotspot' found at codon 249 of exon 7 provides a molecular signature for aflatoxin exposure. ${ }^{1,8}$

From a public health perspective, the combination of hepatitis B vaccination programs and efforts to reduce aflatoxin exposure (Table 4), or to pharmacologically attenuate the toxicological consequences of unavoidable fungal exposure, should have a major impact on the incidence of HCC. ${ }^{3,4}$ The decrease in aflatoxin contamination in the food supply as a result of economic development, coupled to an increase in standard of living has likely contributed, at least in part, to the decreasing burden of HCC in Singapore and Shanghai (China) over the past two decades. ${ }^{3,8,71-73}$

Because aflatoxin contamination in food is not completely avoidable, prevention of aflatoxin-related HCC can also be attempted by the use of agents able to modulate the metabolism of aflatoxin once ingested. Randomized clinical trials of such chemoprevention with oltipraz and chlorophyllin have been conducted in HBsAg-positive carriers exposed to dietary aflatoxin. Both agents modulate levels of aflatoxin biomarkers in the study participants. However, confirmation of the ultimate objective of preventing $\mathrm{HCC}$ would require a large expenditure of resources, and this may not be economically feasible., ${ }^{3,73}$

\section{Avoiding other fungal and algal toxins, and water purity}

In sub-Saharan Africa and China, fumonisin is another fungal toxin that has been linked epidemiologically and mechanistically with liver cancer. Control measures are fundamentally similar to those for aflatoxin, particularly avoidance of moldy corn. In Taiwan, consumption of pond or surface water, particularly that visibly contaminated by algal blooms (green water) was a risk factor for HCC in older studies. ${ }^{3}$ Provision of clean and uncontaminated water is a health priority for the world's poorest communities. Prevention of HCC can be added to the multiple reasons to strive for this basic health objective.

\section{Tobacco smoking awareness}

Tobacco use, the most important preventable cause for many cancers, is only weakly linked to risk for $\mathrm{HCC}^{10,74}$ A Greek study found an interaction between alcohol drinking and tobacco smoking for $\mathrm{HCC}$, after controlling for $\mathrm{HBV}$ and $\mathrm{HCV}$ infec- 
tions. ${ }^{14}$ However, in the presence of stronger risk factors, any possible carcinogenic effect of tobacco is easily masked. Public health and education policies to discourage active and passive smoking are important measures to reduce the incidence of lung and other cancers, but any impact on the risk of HCC is likely to be negligible. ${ }^{75}$

\section{Nutritional modification}

While there is no proven influence of specific nutrients on risk of HCC, micronutrients may play a role..$^{3,76-81}$ Selenium is an essential micronutrient required for selenium-containing anti-oxidant enzymes such as superoxide dismutase. Dietary selenium and retinoic acid are important 'natural' anti-oxidants that inhibit development of liver tumors in animals. ${ }^{76}$ In epidemiological studies of HBsAg carriers from China, selenium deficiency in the water supply and low baseline serum levels of retinol and selenium were associated with increased risk of HCC. Such restoration of these trace elements may be effective chemopreventive strategies., , $^{37-79}$ In addition, diets high in fruits and vegetables or fiber appeared protective against some cancers. ${ }^{3,8,37,38}$ Randomized clinical trials of anti-oxidant-rich dietary supplements, such as selenium, vitamin $\mathrm{A}$, vitamin $\mathrm{C}$, and vitamin $\mathrm{E}$ would be useful in high-risk populations to establish any effect on HCC incidence. ., $, 37,38^{-3}$

The consistent inverse relationship between coffee drinking and HCC is also interesting. ${ }^{80-84}$ Levels of coffee consumption have been associated with reduced risk of cirrhosis irrespective of etiology of liver disease, but it is unclear whether consumption of coffee actually protects against liver injury to reduce risk of HCC. ${ }^{80-84}$ Other explanations are possible. For example, dyspepsia as a side effect of prolonged high blood caffeine levels may lead to dose reductions, as high blood levels likely result from the known impairment of caffeine metabolism that occurs in cirrhosis. ${ }^{85}$ Consumption of green tea is another possible protective factor against HCC. ${ }^{86}$ However, there is currently insufficient evidence to recommend increased consumption of green tea and coffee as preventive measures against $\mathrm{HCC}$.

In summary, HCC is unusual among common human cancers in that one or more causative agents can be identified in most cases. Further, HCC can generally be viewed as a complication of cirrhosis, and the incidence is highest when the factors causing liver injury and inflammation (hepatitis) continue to operate. Although $\mathrm{CHB}$ and/or $\mathrm{CHC}$ are the most important risk factors for HCC worldwide, some other causes of cirrhosis also can lead to HCC. Given the current epidemic of alcoholism and obesity, the incidence of ALD- and NAFLD-related HCC is expected to increase in the future. Therefore, in addition to developing new diagnostic tools and pharmacologic therapies, efforts should also be directed at preventing nonviral liver diseases. To this end, appropriate public health measures should be instituted to combat obesity and diabetes, as well as to target or provide early therapeutic intervention for 'at-risk' individuals before development of cirrhosis. Community-based programs to discourage and deal with excessive alcohol intake, tobacco smoking, prevent exposure to aflatoxin and other toxins, as well as measures aimed at reducing the pandemic of obesity and diabetes and reducing iron overload are logical approaches to interrupt the rising tide of HCC in countries where, until recently, it has not been a common cause of cancer death.

\section{References}

1 El-serag HM, Rudolph KL. Hepatocellular carcinoma: epidemiology and molecular carcinogenesis. Gastroenterology 2007; 132: 2557-76.

2 Yuen MF, Hou JL, Chutaputti A et al. Epidemiology of hepatocellular carcinoma in Asia-Pacific region. J. Gastroenterol. Hepatol. 2009; 24: 346-53.

3 Lodato F, Mazzella G, Festi D et al. Hepatocellular carcinoma prevention: A worldwide emergence between the opulence of developed countries and the economic constraints of developing nations. World J. Gastroenterol. 2006; 12: 7239-49.

4 Hainaut P, Boyle P. Curbing the liver cancer epidemic in Africa. Lancet 2008; 371: 367-8.

5 Koorey D. Hepatocellular carcinoma: prevention, detection and treatment in the real world. Intern. Med. J. 2007; 37: 513-15.

6 Pascual S, Irurzun J, Zapater P et al. Usefulness of surveillance programmes for early diagnosis of hepatocellular carcinoma in clinical practice. Liver Int. 2008; 28: 682-9.

7 Amarapurkar D, Han KH, Chan HLY et al. Surveillance program for hepatocellular carcinoma. J. Gastroenterol. Hepatol. 2009; in press.

$8 \mathrm{Yu}$ MC, Yuan JM. Environmental factors and risk for hepatocellular carcinoma. Gastroenterology 2004; 127: S72-8.

9 Lim SG, Kao KH, Mohamed R et al. Prevention of hepatocellular carcinoma in HBV infection. J. Gastroenterol. Hepatol. 2009; in press.

10 Ueno Y, Sollano J, Farrell GC. Prevention of hepatocellular carcinoma complicating chronic hepatitis C. J. Gastroenterol. Hepatol. 2009; 24: 531-6.

11 Wong R, Corley DA. Racial and ethnic variations in hepatocellular carcinoma incidence within the United States. Am. J. Med. 2008; 121: $525-31$.

12 Velazquez RF, Rodriguez M, Navascues CA et al. Prospective analysis of risk factors for hepatocellular carcinoma in patients with liver cirrhosis. Hepatology 2003; 37: 520-7.

13 Raza SA, Clifford GM, Franceschi S. Worldwide variation in the relative importance of hepatitis $\mathrm{B}$ and hepatitis $\mathrm{C}$ viruses in hepatocellular carcinoma: a systematic review. Br. J. Cancer 2007; 96: $1127-34$.

14 Donato F, Gelatti U, Limina RM, Fattovich G. Southern Europe as an example of interaction between various environmental factors: a systemic review of the epidemiologic evidence. Oncogene 2006; 25 : 3756-70.

15 Paul SB, Sreenivas V, Gulati MS et al. Incidence of hepatocellular carcinoma among Indian patients with cirrhosis of liver: an experience from a tertiary care center in northern India. Indian J. Gastroenterol. 2007; 26: 274-8.

16 Ohishi W, Fujiwara S, Cologne JB et al. Risk factors for hepatocellular carcinoma in a Japanese population: a nested case-control study. Cancer Epidemiol. Biomarkers Prev. 2008; 17: 846-54.

17 Schuppan D, Afdhal NH. Liver cirrhosis. Lancet 2008; 371: 838-51.

18 Ahmad J, Rabinovitz M. Etiology and epidemiology of hepatocellular carcinoma. In: Carr BI, ed. Hepatocellular Cancer: Diagnosis and Treatment. Totowa, NJ: Humana Press Inc., 2005; $1-22$.

19 Stravitz RT, Heuman DM, Chand N et al. Surveillance for hepatocellular carcinoma in patients with cirrhosis improves outcome. Am. J. Med. 2008; 121: 119-26.

20 Deutsch M, Papatheodoridis GV, Tzakou A, Hadziyannis SJ. Risk of hepatocellular carcinoma and extrahepatic malignancies in primary biliary cirrhosis. Eur. J. Gastroenterol. Hepatol. 2008; 20: 5-9.

21 Silveira MG, Suzuki A, Lindor KD. Surveillance for hepatocellular carcinoma in patients with primary biliary cirrhosis. Hepatology 2008; 48: 1149-56.

22 Thompson Coon J, Rogers G, Hewson P et al. Surveillance of 
cirrhosis for hepatocellular carcinoma: a cost-utility analysis. $\mathrm{Br} . \mathrm{J}$. Cancer 2008; 98: 1166-75.

23 Yeoman AD, Al-Chalabi T, Karani JB et al. Evaluation of risk factors in the development of hepatocellular carcinoma in autoimmune hepatitis: implications for follow-up and screening. Hepatology 2008; 48: 863-70.

24 Hashizume H, Sato K, Takagi H et al. Primary liver cancers with nonalcoholic steatohepatitis. Eur. J. Gastroenterol. Hepatol. 2007; 19: 827-34.

25 Bugianesi E. Non-alcoholic steatohepatitis and cancer. Clin. Liver Dis. 2007; 11: 191-207.

26 Sanyal AJ, Banas C, Sargeant C et al. Similarities and differences in outcomes of cirrhosis due to nonalcoholic steatohepatitis and hepatitis C. Hepatology 2006; 43: 682-9.

27 Cuadrado A, Orive A, Garcia-Suarez C et al. Non-alcoholic steatohepatitis (NASH) and hepatocellular carcinoma. Obes. Surg. 2005; 15: 442-6.

28 Bruix J, Forner A, Varmen C, Llovet JM. Primary liver cell carcinoma. In: Rodes J, Benhamou JP, Blei AT, et al., eds. Textbook of Hepatology, 3rd edn. Boston: Blackwell Publishing, 2007; 1437-56.

29 Cook BL, Liesveld J. Alcohol-related health problems. In: Wallace RB, Kohatsu N, eds. Public Health and Preventive Medicine, 15th edn. New York: McGraw-Hill Companies, Inc, 2008; 999-1012.

30 Higuchi S, Matsushita S, Maesato H, Osaki Y. Japan: alcohol today. Addiction 2007; 102: 1849-62.

31 Berry JG, Pidd K, Roche AM, Harrison JE. Prevalence and patterns of alcohol use in the Australian workforce: findings from the 2001 National Drug Strategy Household Survey. Addiction 2007; 102: 1399-410.

32 Vecchia CL. Alcohol and liver cancer. Eur. J. Cancer Prev. 2007; 16: $495-7$

33 McKillop IH, Schrum LW. Alcohol and liver cancer. Alcohol 2005; 35: 195-203.

34 Morgan TR, Mandayam S, Jamal M. Alcohol and hepatocellular carcinoma. Gastroenterology 2004; 127: S87-96.

35 Hassan MM, Hwang LY, Hatten CJ et al. Risk factors for hepatocellular carcinoma: synergism of alcohol with viral hepatitis and diabetes mellitus. Hepatology 2002; 36: 1206-13.

36 Renehan AG, Tyson M, Egger M et al. Body-mass index and incidence of cancer: a systemic review and meta-analysis of prospective observational studies. Lancet 2008; 371: 569-78.

37 Klein S, Romijin JA. Obesity. In: Kronenberg HM, Melmed S, Polonsky KS, Larsen PR, eds. Williams Textbook of Endocrinology, 11th edn. Philadephia, PA: Saunders Elsevier, 2008; 1563-87.

38 Larsson SC, Wolk A. Excess body fatness: an important cause of most cancers. Lancet 2008; 371: 536-37.

39 Qian Y, Fan JG. Obesity, fatty liver and liver cancer. Hepatobiliary Pancreat. Dis. Int. 2005; 4: 173-7.

40 Harrison SA. Liver disease in patients with diabetes mellitus. J. Clin. Gastroenterol. 2006; 40: 68-76.

41 El-Serag HB, Hampel H, JAvadi F. The association between diabetes and hepatocellular carcinoma: a systematic review of epidemiologic evidence. Clin. Gastroenterol. Hepatol. 2006; 4: 369-80.

42 Teoh NC, Fan JG. Diabetes mellitus and prognosis after curative therapy for hepatocellular carcinoma: Alas, still grave for those who are hyperglycemic. J. Gastroenterol. Hepatol. 2008; 23: 1633-4.

43 Fan JG, Farrell GC. Epidemiology of nonalcoholic fatty liver disease in China. J. Hepatol. 2009; 50: 204-10.

44 Fan JG, Saibara T, Chitturi S et al. What are the risk factors and settings of nonalcoholic fatty liver disease in Asia-Pacific. J. Gastroenterol. Hepatol. 2007; 22: 794-800.

45 Farrell GC, Larter CZ. Nonalcoholic fatty liver disease: from steatosis to cirrhosis. Hepatology 2006; 43: S99-112.
46 Allen KJ, Gurrin LC, Constantine CC et al. Iron-overload-related disease in HFE hereditary hemochromatosis. N. Engl. J. Med. 2008; 358: $221-30$.

47 Kowdley KV. Iron, hemochromatosis, and hepatocellular carcinoma. Gastroenterology 2004; 127: S79-86.

48 Beaton MD, Adams PC. Prognostic factors and survival in patients with hereditary hemochromatosis and cirrhosis. Can. J. Gastroenterol. 2006; 20: 257-60.

49 von Delius S, Lersch C, Schulte-Frohlinde E et al. Hepatocellular carcinoma associated with hereditary hemochromatosis occurring in non-cirrhotic liver. Z. Gastroenterol. 2006; 44: 39-42.

$50 \mathrm{Kew}$ MC, Asare GA. Dietary iron overload in the African and hepatocellular carcinoma. Liver Int. 2007; 27: 735-41.

51 Nahon P, Sutton A, Rufat P et al. Liver iron, HFE gene mutations, and hepatocellular carcinoma occurrence in patients with cirrhosis. Gastroenterology 2008; 134: 102-10.

52 Zakhari S, Li TK. Determinants of alcohol use and abuse: impact of quantity and frequency patterns on liver disease. Hepatology 2007; 46: 2032-9.

53 Kotlyar DS, Burke A, Campbell MS, Weinrieb RM. A critical review of candidacy for orthotopic liver transplantation in alcoholic liver disease. Am. J. Gastroenterol. 2008; 103: 734-43.

54 Day CP. Treatment of alcoholic liver disease. Liver Transpl. 2007; 13: S69-75.

55 Torres DM, Harrison SA. Diagnosis and treatment of nonalcoholic steatohepatitis. Gastroenterology 2008; 134: 1682-98.

56 Farrell GC, Chitturi S, Lau GK et al. Guidelines for the assessment and management of non-alcoholic fatty liver disease in the Asia-Pacific region: executive summary. J. Gastroenterol. Hepatol. 2007; 22: 775-7.

57 Chan HL-Y, de Silva HJ, Leung NW-Y et al. How should we manage patients with non-alcoholic fatty liver disease in 2007 ? J. Gastroenterol. Hepatol. 2007; 22: 801-8.

58 Allen KJ. Population genetic screening for hereditary haemochromatosis: are we a step closer? Med. J. Aust. 2008; 189: $300-1$.

59 Phatak PD, Bonkovsky HL, Kowdley KV. Hereditary hemochromatosis: time for targeted screening. Ann. Intern. Med. 2008; 149: 270-2.

60 Allen KJ, Nisselle AE, Collins VR et al. Asymptomatic individuals at genetic risk of haemochromatosis take appropriate steps to prevent disease related to iron overload. Liver Int. 2008; 28: 363-9.

61 Elmberg M, Hultcrantz R, Ekbom A et al. Cancer risk in patients with hereditary hemochromatosis and in their first-degree relatives. Gastroenterology 2003; 125: 1733-41.

62 Fowler C. Hereditary hemochromatosis: pathophysiology, diagnosis, and management. Crit. Care Nurs. Clin. North Am. 2008; 20: 191-201.

63 Bring P, Partovi N, Ford JA, Yoshida EM. Iron over-load disorders: treatment options for patients refractory to or intolerant of phlebotomy. Pharmacotherapy 2008; 28: 331-42.

64 Jackson H, Solaymani-Dodaran M, Card TR et al. Influence of ursodeoxycholic acid on the mortality and malignancy associated with primary biliary cirrhosis: a population-based cohort study. Hepatology 2007; 46: 1131-7.

65 Tarao K, Fujiyama S, Ohkawa S et al. Ursodiol use is possibly associated with lower incidence of hepatocellular carcinoma in hepatitis C virus-associated liver cirrhosis. Cancer Epidemiol. Biomarkers Prev. 2005; 14: 164-9.

66 Gong Y, Huang Z, Christensen E, Gluud C. Ursodeoxycholic acid for patients with primary biliary cirrhosis: an updated systematic review and meta-analysis of randomized clinical trials using Bayesian approach as sensitivity analyses. Am. J. Gastroenterol. 2007; 102: 1799-807. 
67 De Maria N, Manno M, Villa E. Sex hormones and liver cancer. Mol. Cell. Endocrinol. 2002; 193: 59-63.

68 Giannitrapani L, Soresi M, La Spada E et al. Sex hormones and risk of liver tumor. Ann. N. Y. Acad. Sci. 2006; 1089: 228-36.

69 Ming L, Thorgeirsson SS, Gail MH et al. Dominant role of hepatitis $\mathrm{B}$ virus and cofactor role aflatoxin in hepatocarcinogenesis in Qidong, China. Hepatology 2002; 36: 1214-20.

70 Turner PC, Sylla A, Diallo MS et al. The role of aflatoxins and hepatitis viruses in the etiopathogenesis of hepatocellular carcinoma: a basis for primary prevention in Guinea-Conakry, West Africa. J. Gastroenterol. Hepatol. 2002; 17: S441-8.

71 Groopman JD, Kensler TW, Wild CP. Protective interventions to prevent aflatoxin-induced carcinogenesis in developing countries. Annu. Rev. Public Health 2008; 29: 187-203.

72 Turner PC, Sylla A, Gong YY et al. Reduction in exposure to carcinogenic aflatoxins by post-harvest intervention measures in West Africa: a community-based intervention study. Lancet 2005; 365: 1950-56.

73 Kensler TW, Egner PA, Wang JB et al. Chemoprevention of hepatocellular carcinoma in aflatoxin endemic areas. Gastroenterology 2004; 127: S310-18.

74 Marrero JA, Fontana RJ, Fu S et al. Alcohol, tobacco and obesity are synergistic risk factors for hepatocellular carcinoma. J. Hepatol. 2005; 42: 218-24.

75 Britton J, Edwards R. Tobacco smoking, harm reduction, and nicotine product regulation. Lancet 2008; 371: 441-5.

$76 \mathrm{Xu} \mathrm{J}$, Yang F, An X, Hu Q. Anticarcinogenic activity of selenium-enriched green tea extracts in vivo. J. Agric. Food Chem. 2007; 55: 5349-53.
77 Yuan JM, Gao YT, Ong CN et al. Prediagnostic level of serum retinol in relation to reduced risk of hepatocellular carcinoma. J. Natl. Cancer Inst. 2006; 98: 482-90.

78 Sakoda LC, Graubard BI, Evans AA et al. Toenail selenium and risk of hepatocellular carcinoma mortality in Haimen City, China. Int. J. Cancer 2005; 115: 618-24.

79 Chin-Thin W, Wei-Tun C, Tzu-Ming P, Ren-Tse W. Blood concentrations of selenium, zinc, iron, copper and calcium in patients with hepatocellular carcinoma. Clin. Chem. Lab. Med. 2002; 40: $1118-22$.

80 Bravi F, Bosetti C, Tavani A et al. Coffee drinking and hepatocellular carcinoma risk: a meta-analysis. Hepatology 2007 46: $430-5$

81 Larsson SC, Wolk A. Coffee consumption and risk of liver cancer: a meta-analysis. Gastroenterology 2007; 132: 1740-5.

82 Tanaka K, Hara M, Sakamoto T et al. Inverse association between coffee drinking and the risk of hepatocellular carcinoma: a case-control study in Japan. Cancer Sci. 2007; 98: 214-18.

83 Montella M, Polesel J, La Vecchia C et al. Coffee and tea consumption and risk of hepatocellular carcinoma in Italy. Int. J. Cancer 2007; 120: 1555-9.

84 La Vecchia C. Coffee, liver enzymes, cirrhosis and liver cancer. J. Hepatol. 2005; 42: 444-6.

85 Park GJ, Katelaris PH, Jones DB et al. Validity of the 13C-caffeine breath test as a noninvasive, quantitative test of liver function. Hepatology 2003; 38: 1227-36.

86 Jin X, Zheng RH, Li YM. Green tea consumption and liver disease: a systematic review. Liver Int. 2008; 28: 990-6. 